\title{
Anabases
}

ANABASES Traditions et réceptions de l'Antiquité

$18 \mid 2013$

Varia

\section{Richard HUNTER, Plato and the traditions of ancient \\ literature : the silent stream}

Mathilde Cambron-Goulet

\section{OpenEdition}

Journals

Édition électronique

URL : http://journals.openedition.org/anabases/4401

DOI : 10.4000/anabases.4401

ISSN : 2256-9421

Éditeur

E.R.A.S.M.E.

Édition imprimée

Date de publication : 1 octobre 2013

Pagination : 279-281

ISSN : 1774-4296

Référence électronique

Mathilde Cambron-Goulet, "Richard HUNTER, Plato and the traditions of ancient literature : the silent stream », Anabases [En ligne], 18 | 2013, mis en ligne le 01 janvier 2013, consulté le 22 septembre 2020. URL : http://journals.openedition.org/anabases/4401 ; DOI : https://doi.org/10.4000/anabases. 4401

Ce document a été généré automatiquement le 22 septembre 2020

(c) Anabases 


\title{
Richard HUNTER, Plato and the traditions of ancient literature: the silent stream
}

\author{
Mathilde Cambron-Goulet
}

\section{RÉFÉRENCE}

Richard HUNTER, Plato and the traditions of ancient literature : the silent stream, Cambridge et New York, Cambridge University Press, 2012, 279 p.

99,00 dollars / ISBN 9781107012929.

1 Le professeur Richard Hunter, Regius Professor of Greek à l'université de Cambridge, tente, avec cet ouvrage très érudit, une réappropriation dans le champ littéraire de l'œuvre de Platon, trop souvent abandonnée aux philosophes. Il s'agit donc d'étudier les divers intertextes platoniciens: intertexte poétique et littéraire dans l'œuvre de Platon et intertexte platonicien dans les œuvres poétiques et littéraires postérieures.

2 Hunter commence par s'attacher à la dimension culturelle des textes platoniciens comme marqueurs de l'identité grecque. C'est l'objet du premier chapitre, «Introduction: Tracing Plato ». Platon, comme Homère, devient ainsi une pierre angulaire de la culture grecque, et trouve des échos dans la littérature subséquente dans des formes complexes qui peuvent se comparer à des allusions poétiques (p. 22).

3 Le deuxième chapitre, «Homer and Plato ", est consacré à la dette de Platon à l'endroit d'Homère, telle qu'elle était perçue chez les critiques littéraires de l'Antiquité. Pour ce faire, il examine l'intertexte homérique qui traverse l'œuvre de Platon, et comment les lectures platoniciennes d'Homère font partie, à leur tour, de l'intertexte de la littérature grecque postérieure. Il montre, de manière convaincante, que Platon faisait davantage partie du paysage littéraire que philosophique : «the critical tradition has turned Plato's powerful intellectual challenge to Homer into a literary and stylistic competition with him. » (p. 43). 
4 Le chapitre 3, "Metamorphoses of the Apology", est organisé autour de l'Apologie de Socrate, et porte sur le rapport entre l'Apologie et les textes d'orateurs contemporains (Lysias, Démosthène, Isocrate); il montre que l'Apologie ne s'éloigne que peu des normes des plaidoyers de défense (p. 116). Hunter s'attache ensuite à en retrouver les motifs dans la littérature subséquente, en examinant entre autres le thème des interventions oraculaires dans les vies de philosophes (p. 120).

5 Le chapitre 4, «Dionysius of Halicarnassus and the style of the Phaedrus », est consacré aux considérations de la critique littéraire antique sur le style du Phèdre. Hunter montre habilement, en passant par la réception que font Longin, Hermeias et Favorinus de l'œuvre de Platon, comment Denys d'Halicarnasse confronte deux lieux communs de

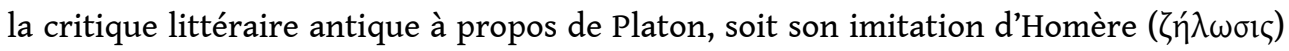

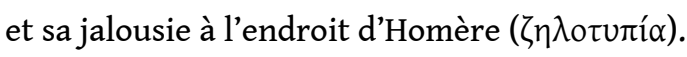

6 Le chapitre 5, "Plato as classic: Plutarch's Amatorius ", ne se contente pas de montrer les parallèles entre l'ouvrage de Plutarque et les différents motifs platoniciens du Phèdre et du Banquet, mais fait intervenir le passage par le roman grec. Même si les emprunts de Plutarque à Platon sont évidents dans la mesure où le sujet et la forme sont platoniciens, Hunter tire parti de ce thème pour étudier comment Platon peut être opposé à Platon, car les protagonistes du Dialogue sur l'amour parviennent toujours à placer Platon de leur côté dans le débat.

7 Le dernier chapitre, "Playing with Plato", montre des ouvrages anciens qui empruntent à Platon ses motifs narratifs plutôt que sa pensée. Ainsi, des auteurs de romans latins (Apulée, Pétrone) se figurent partager des modèles poétiques avec Platon (p.225). Cette culture partagée s'exprime notamment par le mélange de sérieux ( $\left.\sigma \pi \circ v_{\delta \eta}\right)$ et d'amusant ( $ү \varepsilon \lambda$ oĩos). Cependant, Hunter nous convainc de la véritable présence de Platon dans ces ouvrages puisque les auteurs empruntent également à Platon certains thèmes, par exemple la question de la vérité du discours (p. 241).

8 L'ouvrage de Richard Hunter se présente ainsi comme une série d'études de cas avec en arrière-plan trois objectifs ambitieux. D'une part, l'auteur souhaite montrer comment Platon engage un dialogue non seulement philosophique mais aussi stylistique avec les discours poétiques ou prosaïques qui lui sont antérieurs ou contemporains. D'autre part, Hunter examine les discours antiques sur le style platonicien, et la manière dont la critique littéraire antique le compare aux styles d'autres auteurs. Enfin, il nous invite à voir dans les discours platoniciens des marqueurs identitaires du monde grec (p. 12), et étudie par conséquent les différentes imitations du style et des arguments de Platon dans la littérature antique subséquente. Les trois objectifs superposés, qui font sans doute la richesse du volume non seulement pour les littéraires mais aussi pour les philosophes, le rendent peu accessible malgré la clarté du style, car l'étude de l'intertexte des dialogues platoniciens traité par des couches successives d'auteurs plus tardifs crée un effet d'emboîtement à la manière des poupées russes. Ces emboîtements provoquent un certain nombre de dédoublements (par exemple Longin, Du sublime 13.2, dont on retrouve les racines dans l'Ion à la p. 43 et dans le Banquet à la p.127), compensés par un index des passages cités assez complet. Enfin, l'ouvrage de Hunter soulève un certain nombre de problèmes inhérents au sujet, que l'auteur reconnaît volontiers : l'influence de Platon sur des orateurs contemporains est difficile à établir, car les parallèles pourraient relever d'une culture commune plutôt qu'être dus à des emprunts (p. 27) ; plus on avance dans l'Antiquité, plus Platon fait partie de la culture commune, et plus il devient difficile de différencier les emprunts directs à Platon des 
emprunts indirects (p. 223). Malgré ce problème de topos, Hunter réussit, grâce à un important travail d'érudition, à nous convaincre du bien-fondé de son entreprise, car si des incertitudes subsistent à propos de certains passages, l'accumulation de preuves montre la prégnance des œuvres platoniciennes et du discours savant sur les œuvres platoniciennes et leur intertexte dans la littérature antique.

\section{AUTEURS}

\section{MATHILDE CAMBRON-GOULET}

Université Paris 7 Diderot / UMR 8210

mathilde.cambron-goulet@umontreal.ca 